\title{
Retrospective audit of the efficacy and safety of the combined intranasal/intravenous midazolam sedation technique for the dental treatment of adults with learning disability
}

IN BRIEF

- This technique can be safely and effectively used in primary care and to treat adults with learning disability.

- The option of providing repeat review examinations and a continuing course of treatment, for example endodontics or periodontal therapy, is possible using this technique.

- The technique allows for an oral exam and diagnosis within primary care rather than immediate referral for general anaesthetic.

\author{
M. C. G. Manley, ${ }^{1}$ N. J. Ransford, ${ }^{2}$ D. A. Lewis, ${ }^{3}$ S. A. Thompson ${ }^{4}$ \\ and M. Forbes ${ }^{5}$
}

Introduction The provision of dental care for adults with severe learning disability presents problems. The approach to treatment has often been provided under general anaesthesia tending to result in exodontia rather than restorative care. This paper presents an alternative to this option using conscious sedation. Methods A multi-centred retrospective audit was reported on using data from Canterbury, Warwick, Dorset and Cardiff. Patients included adults with varying degrees of disability for whom treatment using local anaesthesia, inhalation sedation, and the acceptance of intravenous cannulation was not possible. Sedation was provided by midazolam first administered by the intranasal followed by the intravenous route. Results From a total of 222 episodes of sedation 128 (57.65\%) accepted treatment well and 75 (33.78\%) presented slight problems which did not compromise treatment. In 19 cases (8.55\%) treatment with sedation was not possible and a referral was made for general anaesthesia. Conclusions Results showed that a range of different treatments were carried out including advanced restorative care. This paper proposes that the technique described is safe and effective in providing a good standard of dental care for adults with severe learning disability.

\section{INTRODUCTION}

For people with a disability the ideal provision of oral health care is from practitioners in the primary care setting. However, for certain groups and particularly those with severe learning disability, their care may be more appropriately provided by practitioners with special skills and facilities ${ }^{1}$ such as those working in the Salaried Primary Care and Hospital Services. For some people with disability the continuing management of

1*5.5ental Department, Canterbury Health Centre, 26 Old Dover Road, Canterbury, Kent, CT1 3JH; ${ }^{2}$ Dental Department, Whitnash Lodge, Rehabilitation Hospital, Heathcote Lane, Warwick, CV34 6SR; ${ }^{3}$ Canford Health Centre, Culliford Crescent, Poole, Dorset, BH17 9DW ${ }^{4}$ Division of Adult Dental Health, School of Dentistry, Cardiff University, Cardiff, CF14 4XY

${ }^{*}$ Correspondence to: Dr M. Graham Manley

Email: graham.manley@ekentmht.nhs.uk

\section{Online article number E3}

Refereed Paper - accepted 14 November 2007

DOI: $10.1038 /$ sj.bdj.2008.521

${ }^{\circ}$ British Dental Journal 2008: 205: E3 oral health including periodontal care, routine examinations, and the provision of restorative treatment is very difficult. The approach most often used has been to extract carious or symptomatic teeth under general anaesthetic rather than restorative treatment. This may have contributed to the well documented poorer oral health and dental care outcomes for this group as compared to the general population..$^{2-4}$ As an alternative to this radical and limited approach, the use of intravenous sedation provided in primary care as an operator sedationist has been shown to be an effective management strategy for people with disability. ${ }^{5,6}$ The specific technique of using oral or intranasal midazolam to allow cannulation followed by intravenous sedation of the same drug has made available the option for comprehensive care. This would include effective routine examinations, periodontal care, and restorative treatment. Refinement of the

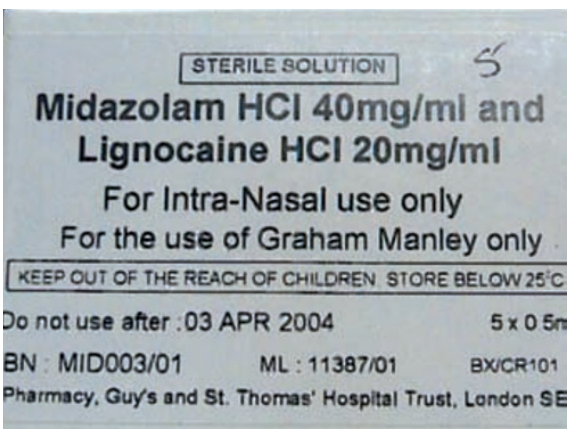

Fig. 1 Concentrated Midazolam previously restricted to one prescriber now freely available by order from Pharmacy Production St Thomas' Hospital

technique of administering midazolam orally and or intranasally by the concentration of midazolam to $40 \mathrm{mg} / \mathrm{ml}$ (Fig. 1) and the inclusion of lidocaine to reduce the stinging effect, has played a significant part in influencing the increasing use of this strategy by practitioners working in the primary care as well as hospital services. ${ }^{7}$ This formulation is better tolerated by patients than 


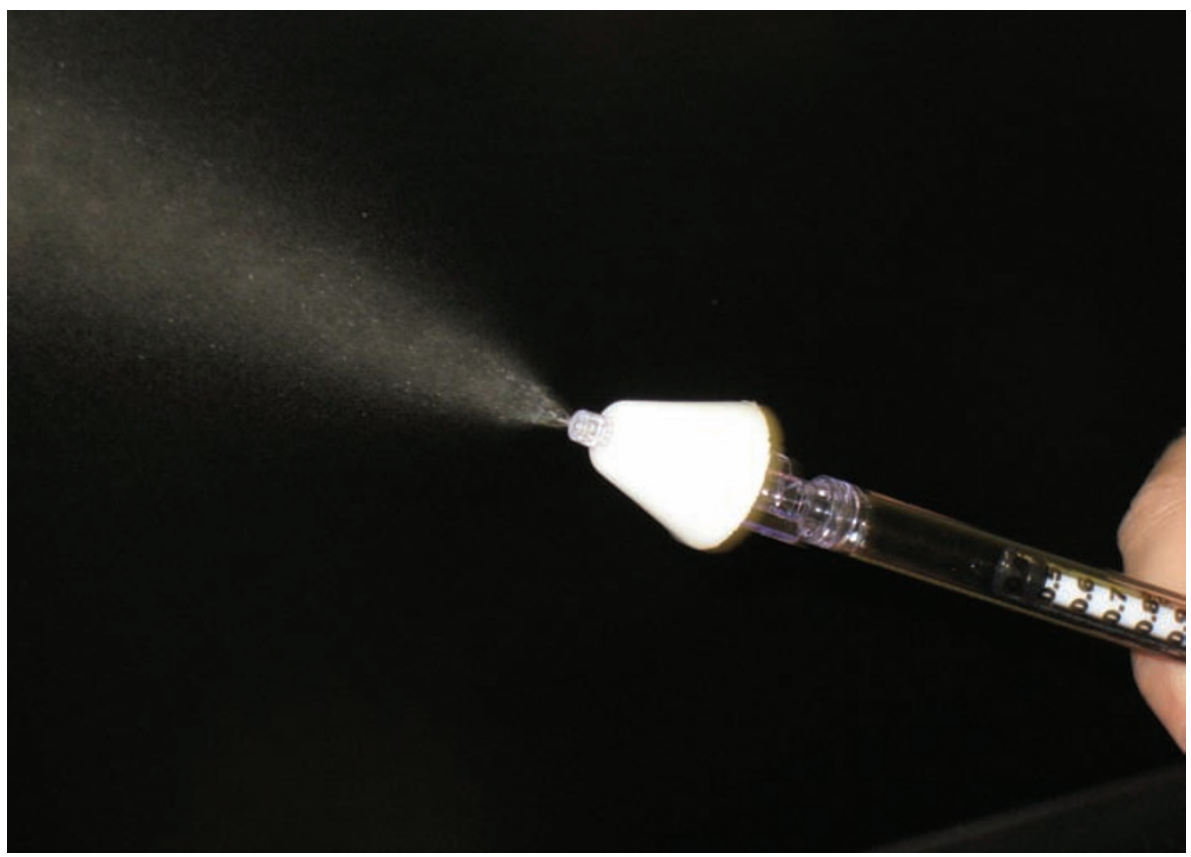

Fig. 2 The Mucosal Atomisation Device connected to a $1 \mathrm{ml}$ syringe

the $10 \mathrm{mg}$ in $2 \mathrm{ml}$ formulation since the concentrated midazolam only requires $0.25 \mathrm{ml}$ volume to deliver a standard 10 $\mathrm{mg}$ dose. The use of the Mucosal Atomising Device ${ }^{8}$ for the production of a fine aerosol (Fig. 2) has further improved the ease and effectiveness of the process of clinical administration.

\section{AUDIT}

As a result of the increasing use of this technique it was considered important to examine its effectiveness and whether any problems had been encountered. A two stage audit cycle was planned with an initial examination of basic information obtained from a large number of cases.This would then be followed by gathering more detailed information in order to be able to further evaluate the technique. In addition it was intended that implementation of this process of the audit cycle would enable the provision of a more helpful protocol to act as a guidance for other colleagues. The retrospective examination of data provided by a group of clinicians, however, presents certain difficulties. The lack of standardisation in clinical procedure, operative criteria for success, data collection, analysis and other variables will inevitably affect the results presented. Conversely advantages can be gained from an initial stage multi-centred audit process. A useful number of cases can be provided enabling a broad base of patients for auditing. From an initial audit much can be learned from different clinicians sharing experiences using a relatively new technique in varying environments. Standards, protocols, and procedures can then be developed with enhanced validity for wider clinical use.

\section{Audit design}

Lead clinicians from the four geographical areas of East Kent, Dorset, Warwickshire and Cardiff were included in the audit. These sites also agreed to take part in the second stage of the audit cycle which will be carried out prospectively and reported on in the future. Approval from the Dental Services Group Clinical Governance Committee of one site was obtained and granted for the audit to be conducted in all four sites. ${ }^{9}$ The initial retrospective audit undertaken obtained data from treatment carried out over a four year period. Data were provided from one experienced clinican/sedationist in the Warwickshire and Cardiff locations, and two clinicians from Dorset. In East Kent data were available from in total 11 clinicians. The East Kent clinicians were of varying levels of experience and expertise. It should, however, be clarified that the inexperienced clinicians operated with careful and close support. They had completed training and were competent in the provision of intravenous sedation using midazolam.
Total number of episodes of sedation from all sources (240)

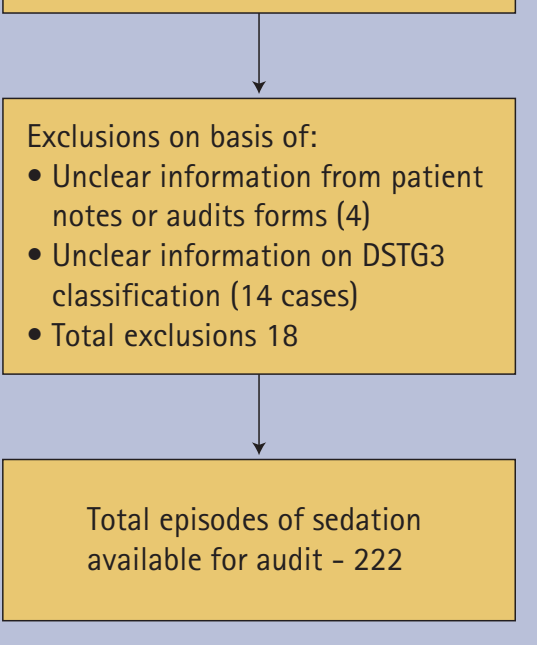

Fig. 3 The process of patient inclusion/ exclusion into the audit

\section{Data collection}

Information was included from the treatment of adults who had received intranasal midazolam which was followed as required by intravenous midazolam. Those included had varying degrees of learning disability and would not tolerate intravenous cannulation. Data were excluded where midazolam had been given prior to general anaesthesia. In assessing the effectiveness of the overall sedation process (ie intranasal and if required intravenous administration) to enable the provision of treatment, the data were analysed from all four sites based on a modified Dental Sedation Teachers Group Scale of operative conditions: ${ }^{10} 1$ - Good: patient fully cooperative; 2 - Fair: minimal interference from patient; 4 - Impossible, needs general anaesthesia to complete treatment. The DSTG category 3 - operating difficult, physical intervention required, was not used since it was considered that it would be too difficult to obtain any sort of consistency in examining the retrospective data from all four sites. Therefore data on 14 cases that were managed and treatment completed with difficulty requiring some physical intervention were excluded. In East Kent and Warwickshire data were extracted from audit forms. Dorset and Cardiff extracted their data from dental records applying the modified DSTG scale. Data were excluded from analysis if there 
was any doubt about judgements made from examining patients' notes. Treatment that was recorded either on the audit forms or patients' notes provided broad based information as to whether examination, periodontal treatment (scaling), restorations, or extractions were completed successfully. Figure 3 provides a flow chart of the process of selection/inclusion into the audit. An assessment of safety of the clinical procedure was obtained from qualitative data available on each case with particular reference to any recorded untoward events of care.

\section{Clinical technique}

All patients were seen initially for assessment and referred for sedation where there was a positive clinical indication at least for an examination and in some cases an obvious provision of treatment. A standard agreement to treat procedure was undertaken and the confirmatory written agreement based on consent form 4 from the Department of Health ${ }^{11}$ was obtained from the relevant carer and a second clinician where appropriate. The outline of treatment clearly stated that intranasal sedation was to be used and information was given regarding off-licence use. A full medical history was obtained and all those treated were in category ASA I or ASA II. ${ }^{12}$ Written pre and post operative instructions were given to the carer at the assessment appointment. On attending for treatment the procedure was outlined to the carers and it was confirmed that they were fully aware of their post operative responsibility. Medical history and agreement to treat were again confirmed, and a blood pressure was recorded if possible. The intranasal midazolam was then administered as a one bolus dose into one nostril with calm support and encouragement. The patient was then clinically monitored and when judged to be sufficiently relaxed and sedated to allow placement of a pulse oximeter and then to obtain venous access, cannulation was undertaken. The intravenous midazolam was titrated against the patient's response according to a standard regime. Once a suitable end point was reached treatment was commenced. After completion of treatment the patient once recovered

\section{Table 1 Frequency of Sedation episodes by numbers of patients}

\begin{tabular}{l|l|l}
\hline Numbers of patients & Numbers of sedation episodes & Total numbers of episodes of sedation \\
\hline 94 & 1 & 94 \\
\hline 28 & 2 & 56 \\
\hline 12 & 3 & 36 \\
\hline 2 & 4 & 8 \\
\hline 0 & 5 & 0 \\
\hline 1 & 6 & 6 \\
\hline 2 & 7 & 14 \\
\hline 1 & 8 & 8 \\
\hline Total patients 140 & & Total episodes 222
\end{tabular}

Table 2 Distribution of care provided; total 222

\begin{tabular}{|c|c|c|c|}
\hline $\begin{array}{l}\text { Types of } \\
\text { care provided }\end{array}$ & $\begin{array}{l}\text { DSTG 1- } \\
\text { Good }\end{array}$ & $\begin{array}{l}\text { DSTG } 2 \text { - } \\
\text { Fair }\end{array}$ & $\begin{array}{l}\text { DSTG 4- } \\
\text { Not possible } \\
\text { Refer for GA }\end{array}$ \\
\hline $\begin{array}{l}\text { Exam no } \\
\text { treatment required }\end{array}$ & 6 & $\begin{array}{l}2+1 \text { intranasal only } \\
\text { (total } 3 \text { ) }\end{array}$ & \\
\hline $\begin{array}{l}\text { Exam, radiographs, } \\
\text { scale }\end{array}$ & 7 & 7 & \\
\hline Impressions only & & 2 & \\
\hline Scale and polish & $\begin{array}{l}35+2 \text { blood taken } \\
\text { (total } 37 \text { ) }\end{array}$ & 29 & \\
\hline $\begin{array}{l}\text { Scale and Polish } \\
+ \text { conservation }\end{array}$ & 14 & 2 & \\
\hline $\begin{array}{l}\text { Scale and polish } \\
\text { +extraction }\end{array}$ & 10 & 4 & \\
\hline $\begin{array}{l}\text { Scale and polish } \\
+ \text { conservation+ } \\
\text { extraction }\end{array}$ & 5 & 3 & \\
\hline $\begin{array}{l}\text { Conservation } \\
+ \text { extraction }\end{array}$ & 1 & & \\
\hline Extraction only & $\begin{array}{l}20+2 \text { surgicals } \\
\text { (total 22) }\end{array}$ & $\begin{array}{l}13+2 \text { intranasals only } \\
+1 \text { surgical (total } 16)\end{array}$ & \\
\hline Conservation only & $\begin{array}{l}17+1 \text { blood taken }+4 \\
\text { endodontics } \\
+3 \text { veneers } \\
+1 \text { bridge cementation } \\
\text { (total } 26 \text { ) }\end{array}$ & $\begin{array}{l}6+3 \text { endodontics } \\
\text { (with intranasal only) } \\
\text { total } 9\end{array}$ & \\
\hline Total episodes & $128(57.65 \%)$ & 75 (33.78\%) & $19(8.55 \%)$ \\
\hline
\end{tabular}

\section{Table 3 Frequency of doses of intravenous midazolam}

\begin{tabular}{l|l|}
$\begin{array}{l}\text { Range of dose of intravenous midazolam } \\
\text { administered }\end{array}$ & $\begin{array}{l}\text { Episodes of administration of midazolam } \\
\text { within dose range }\end{array}$ \\
\hline $1-5 \mathrm{mg}$ & 53 \\
\hline $6-10 \mathrm{mg}$ & 109 \\
\hline $11-15 \mathrm{mg}$ & 18 \\
\hline $16-20 \mathrm{mg}$ & 17 \\
\hline $\begin{array}{l}\text { Number of episodes of intravenous midazolam } \\
\text { available from the total audit of 222 }\end{array}$ & $\begin{array}{l}197 \\
19 \text { excluded as referred for general anaesthesia, } \\
\text { six excluded as administered intranasal only }\end{array}$ \\
\hline
\end{tabular}


was discharged into the care of the escort with the appropriate verbal and written instructions.

\section{RESULTS}

A total number of 140 patients were included in the audit. Of these patients some received one episode of sedation only (ie 94 patients) and others received varying numbers of sedation episodes. Table 1 illustrates the numbers of patients that received varying sedation episodes. From these patients 222 episodes of sedation were included in the audit. Of this total 128 (57.65\%) were fully cooperative - DSTG 1.

Seventy-five $(33.78 \%)$ presented minimal interference - DSTG 2 and in a small number of cases, 19 (or 8.55\%) - DSTG 4 any form of care was impossible and a referral was made for treatment under general anaesthetic. Table 2 shows a summary of these results including the combination of different types of treatments that were carried out.

For a small number of cases (6) intranasal sedation alone was used as sufficient sedation was provided to allow for completion of treatment without additional intravenous titration. In three cases in addition to dental care, a blood sample was taken although information was unavailable as to whether the indication for this was following a request from the patients medical practitioner or an investigation was required relating to dental treatment (eg INR).

An accurate record of the drug dosages was not available from all the data due to recording variations. However, where reliable and consistent data were obtained it was shown that in all but 15 cases (where an intranasal dose of over $15 \mathrm{mg}$ was used) the range of intranasal dose was from 4-15 mg with the average $8.3 \mathrm{mg}$ and the mode being $10 \mathrm{mg}$.

The intravenous dosages that were given following the intranasal administration varied and Table 3 shows the frequency distribution of intravenous dosages.

For those patients that were referred for treatment under general anaesthetic this was due to a variety of reasons. These included refusal to accept intranasal administration, resistance to cannulation and/or treatment following intravenous administration.
One episode only of an untoward event relating to the administration of midazolam was recorded. In this case an unusual response of desaturation occurred following the initial administration of intravenous midazolam. This was corrected by the administration of supplemental oxygen.

\section{DISCUSSION}

The value of using this specific sedation technique by a number of clinicians with varying experience was shown from the results. In the majority of episodes of its use (57.65\%) completion of necessary treatment was achieved without difficulty (DSTG 1). This included examination and a variety of aspects of dental care. Table 2 shows that not only were conservation, periodontal care and extractions (including three episodes of surgical extraction) provided but also a combination of these different types of care during each treatment episode. This indicates an efficient use of appointments. In addition in some cases advanced restorative care was also provided including endodontics (seven episodes), use of veneers (three episodes) and provision of a bridge (one case). For those cases where some slight difficulty in completing dental care was experienced (DSTG 2) there was a reduced number of episodes of conservation ie nine episodes compared with 26 episodes in DSTG 1. However, for periodontal care and extractions there was much less difference between good and fair operating conditions. A number of cases (19) needed referring for treatment under general anaesthetic and it must be accepted that as with any clinical technique $100 \%$ success is rarely possible. For that reason this sort of technique must never be proposed as an alternative to general anaesthesia which is in itself a safe and valuable treatment option. ${ }^{13}$ In 66 episodes of sedation (29.72\%) examination and scaling only were provided. This aspect of care is particularly important for adults with disability for whom periodontal disease is a major problem and for which the repeated use of general anaesthesia would not be appropriate. It may have been the case that for some of these patients no examination was possible prior to treatment and an appointment was booked for examination and provision of necessary dental care. For these cases the option of this technique within the primary care environment has several advantages to provision of general anaesthesia in hospital. It is cheaper, more accessible and more acceptable for both the patient and carer. It is important to note that in terms of safety out of the total of 222 episodes of using this technique only one episode of significant desaturation was recorded which responded with administration of oxygen. In the majority of cases an intranasal dose of $10 \mathrm{mg}$ was used, and found to be effective. This is a fairly arbitrary dose based on the bioavailability of intranasal midazolam of approximately $70 \%{ }^{14}$ which would equate to an approximate maximum bolus of $7 \mathrm{mg}$. The actual dose is very likely to be less as a result of ineffective deposition of some midazolam in the intranasal administration process. This use of midazolam off licence presents no problem for the professional clinician who is permitted to use a drug off licence in accordance with clinical need, supported by evidence and experience. ${ }^{15,16}$ The dosages of intravenous midazolam tend to comply with the natural distribution curve (Table 3) with 6-10 mg being the highest frequency group.

Judgement of the DSTG category 3 presented difficulty when examining data from four different sources, the sedation of which had been provided by clinicians of varying experience. For example a difficult patient who could have had all treatment completed with relative ease by a clinician experienced in the management of very difficult cases as well as the use of this sedation technique could be classified as DSTG 2. A clinician with less experience and skill may have referred the case for general anaesthetic (DSTG 4) without being able to undertake any form of treatment. In order to reduce the level of variability and to help provide more consistent data, those patients that presented these sorts of problems in classification (14 in number - Fig. 1) from DSTG 3 were therefore excluded. It could be suggested that because of this there was a bias towards including only the less difficult patients in the audit and this would 
imply a limited value of the technique. However, the proportion excluded was small enough to have a minimal bias on the results. In addition this point merely confirms the fact that, as with all aspects of the provision of dental care, the more skilled and experienced practitioner may achieve more. It should also be remembered that all patients that were included in this audit presented difficulties and problems in management otherwise they would not require intranasal and intravenous sedation. The alternative would have been to refer such patients for general anaesthetic.

\section{CONCLUSION}

This audit shows how a practical technique has developed in response to a clinical need that has been identified in primary care. The robust nature of this technique was confirmed from its safety and effectiveness and to this end provides some valuable data.

The care of people with disability requires the ability to adopt a flexible and innovative approach enabling the management of often unusual and challenging situations.The unswerving adherence to restrictive protocols may in certain circumstances result in a reduced level of care (ie so called protocol paralysis). It is, however, necessary to produce guidance for new and innovative types of clinical techniques both to encourage and to support practitioners who are unfamiliar with the use of sedative drugs in this way and to demonstrate the considerable benefit of this technique for people with learning disability. The technique in itself is not difficult, essentially requiring an initial good understanding and sound experience in the use of intravenous midazolam. It is not nor should it be considered as an advanced technique, ${ }^{17,18}$ and does not involve the use of different sedative drugs. It should be an essential tool for the practitioner who is committed to providing a good quality range of care for people with disability. Consideration must, however, be given to the complexity in management of patients with severe disability such as those described in this audit. It would be strongly advised that practitioners who may be proposing to undertake these sedation techniques should have significant experience both in the use of intravenous conscious sedation and in the provision of dental treatment for people with severe disability. It is very difficult to conceive how a oral health care service for people with disability can provide a comprehensive standard of care without the use of such sedation techniques as described in this audit.

The development and subsequent distribution of Midazolam concentrated at $40 \mathrm{mg} / \mathrm{ml}$ was undertaken by the Pharmacy Production Department at St Thomas' Hospital. The authors are extremely grateful for the support and encouragement of $\mathrm{Mr}$ Paul Forsey and staff who were instrumental in the development of the concentrated midazolam formulation
1. A commissioning tool for special care dentistry. British Society of Disability and Oral Health, 2006

2. Tiller S, Wilson K I, Gallagher J E. The dental health and dental services of adults with learning disabilities. Community Dent Health 2001; 18: 167-171.

3. Steele J G, Sheiham A, Marcenes W, Walls A W G. National diet and nutrition survey adults aged 65 and over. London: HMSO, 1998.

4. Lawton L. Providing dental care for special patients - tips for the general dentists. J Am Dent Assoc 2002; 133: 1666-1670.

5. Manley M C G, Skelly A M, Hamilton A G. Dental treatment for people with challenging behaviour: general anaesthesia or Sedation? Br Dent J 2000; 188: 358-360.

6. Boyle C A, Manley M C G, Fleming G J P. Oral midazolam for adults with learning disabilities. Dent Update 2000; 27: 190-192.

7. List of sources of use of concentrated Midazolam. Personal Communication. St Thomas' Department of Pharmacy Production, 2006.

8. The Mucosal Atomisation Device Intavent Orthofix, Burney Court, Cordwallis Park Maidenhead, Berks SL6 7BZ.

9. Personal Communication. Cardiff and Vale NHS Trust Dental Services Group. Clinical Governance Committee.

10. Assessment of Operating Conditions. Logbook of Clinical Experience in Conscious Sedation. Dental Sedation Teachers Group. www.dstg.co.uk.

11. Department of Health. Good practice in consent implementation guide: consent to examination or treatment.

12. American Society of Anesthesiologists. New classification of physical status. Anesthesiology 1963; 24: 111.

13. Jenkins K, Baker A B. Consent and anaesthetic risk. Anaesthesia 2003; 58: 962-984.

14. Wermeling D P, Record K A, Archer S M, Rudy A C. Pharmacokinetics and pharmacodynamics of a new intranasal midazolam formulation. Anesth Analg 2006; 103: 344-349.

15. Pickles $H$. The use of unlicensed drugs. Br J Healthc Manage 1996; 2: 656-658.

16. British National Formulary, September 2006

17. Commissioning Conscious Sedation in Primary Dental Care. Department of Health February, 2007.

18. Faculty of Dental Surgery. The Royal College of Surgeons of England, The Royal College of Anaesthetists Standards for Conscious Sedation in Dentistry: alternative techniques. A Report from The Standing Committee on Sedation in Dentistry, August 2007. 